\title{
Kritische Frömmigkeit
}

\section{Pierre Bühler}

Kann man heute überhaupt noch von „Frömmigkeit“ sprechen? Das Wort scheint überholt, abgetan, und es besteht wenig Hoffnung, es neu aufwerten zu können. Es lässt gleich negative Assoziationen aufkommen, wie etwa in folgenden Ableitungen: frömmeln, Frömmler, Frömmelei, frömmlerisch. So bezeichnen denn auch in spontanem Verständnis die Wörter "fromm" und „Frömmigkeit" religiöse Einstellungen, die eher wirklichkeitsfremd, in sich selbst verschlossen, wenig gesprächsfähig sind, und deshalb das genaue $\mathrm{Ge}-$ genteil von Freiheit evozieren.

Von diesem Verständnis her beurteilt scheint die Verbindung, die meine Überschrift andeutet, schlechterdings absurd: wenn es so etwas wie Frömmigkeit überhaupt noch gibt, dann ist sie nicht kritisch, sondern viel eher unkritisch. Für sie ist kritisches Denken gefährlich, bedrohend, und deshalb ist sie nicht bereit, sich auf die kritischen Infragestellungen des Denkens einzulassen, siedelt sich lieber jenseits des Denkens an.

Trotzdem möchte ich es versuchen, gerade durch diese anscheinend fragwürdige Verbindung einen neuen Zugang zum Thema Frömmigkeit zu finden. Doch zunächst soll die Berechtigung dieses Versuchs noch etwas genauer erörtert werden.

\section{Faszination der Spiritualitäten}

Es gibt auch heutzutage - in vielerlei Gestalt - enge, weltfremde, verschlossene Frömmigkeit. Sie ist in religiösen Kreisen zu finden, die sich radikal gegen alles Weltliche abgrenzen. Diese Formen von Frömmigkeit bestätigen das soeben erwähnte negative Vorurteil. Darin liegt der erste Grund für die Bemühung um eine erneuerte Auffassung: Frömmigkeit ist eine allzu ernste Sache, um sie einfach den Frömmlern zu überlassen! Doch ein zweiter Grund kommt hinzu: auch bei solchen, die Frömmigkeit als veraltet verwerfen, bleibt anscheinend ein Interesse, freilich umorientiert, umgestaltet. Es ist in diesem Sinne auffallend, wie stark sich heute bei schwindender Frömmigkeit die Faszination für allerlei Spiritualitäten manifestiert, von Zen-Buddhismus über Transzendentale Meditation bis zu New Age oder parapsychologische Erfahrungsgruppen! Das Exotische wirkt anziehend, Auswege aus der Enge der alten Frömmigkeit anbietend. Doch ist darin nicht auch ein Zeichen dafür enthalten, dass das Thema Frömmigkeit nicht bereits erledigt ist? Dass sich in der Attraktivität der Spiritualitäten die Suche nach einem Ersatz für die verlorene Frömmigkeit manifestiert?

Deshalb scheint es mir sinnvoll, das Problem zu reflektieren, zwischen verengtem Traditionalismus und verschwommenem Exotismus. 


\section{Zurück zum ursprünglichen Sinn}

Wie oft in hermeneutischer Perspektive lohnt sich ein kurzer Blick auf die Begriffsgeschichte. Es ist zu bemerken, dass ,fromm“ ursprünglich nicht spezifisch religiös bestimmt war. Das Adjektiv geht vom althochdeutschen Substantiv fruma aus, das „Nutzen, Vorteil, Wohl“ heisst. Dieser alte Sinn ist auch noch in der etwas altertümlichen Formel „zu Nutz und Frommen“ wiederzuerkennen. Auch wird das Verb „frommen" im Sinne von ,nutzen“ gebraucht.

Man könnte also sagen, dass die Frage der Frömmigkeit folgende ist: was frommt dem Menschen? Was nützt ihm? Natürlich gilt diese Frage nicht einfach in einem utilitaristischen Sinne. Sie ist als Frage zu verstehen, die alles danach beurteilt, was es für das Leben des Menschen austrägt. Darin liegt durchaus ein kritisches Prinzip, auch dem Denken gegenüber. Frömmigkeit vollzieht die Verbindung zur Lebenserfahrung, und in theologischer Hinsicht zum gelebten Glauben als dem entscheidenden, kritischen Punkt in der theologischen Sache überhaupt. Es wäre hier also von einer kritischen Frömmigkeit zu sprechen, weil sie alle menschlichen Tätigkeiten, ob denkerisch oder handlungsorientiert, immer wieder auf das Lebensentscheidende hinweist.

Doch wie sieht dieses Lebensentscheidende aus? Ich möchte es im folgenden an einigen Merkmalen näher charakterisieren.

\section{Existentielle Rechtschaffenheit}

Von der bereits erwähnten Ursprungsbedeutung her hiess das Adjektiv „fromm“" zuerst „nützlich", um dann mehr und mehr eine ethische Betonung zu bekommen, im Sinne von „tüchtig, tapfer, rechtschaffen“. Diesen Aspekt möchte ich hier in existentieller Ausrichtung aufnehmen. Die Frage nach dem, was dem Menschen frommt, drückt die Sorge um die Grundausrichtung des Lebens aus: Wer bin ich? Was macht mich zu dem, der ich bin? Wo liegt der Gehalt, der Sinn, der Ernst meines Lebens, meines Glaubens? Was macht aus mir einen rechtschaffenen, mit sich selbst im Einklang lebenden, einen von sich selbst zu sich selbst befreiten Menschen? Das sind Fragen, die die Herausforderung der Frömmigkeit in einem auf das Leben bezogenen, existentiellen Sinne zum Ausdruck bringen. Es geht hier darum, den Menschen immer wieder darauf hinzuweisen, dass er aufgerufen ist, wahrlich er selbst zu sein. Freilich muss aber zugleich die umgekehrte Dimension betont werden.

\section{Öffnung nach aussen hin}

Frömmigkeit wird oft, wie wir bereits gesehen haben, als verschlossene Einstellung abgetan. Doch muss hier genau umgekehrt argumentiert werden: wahre Frömmigkeit schafft Öffnung nach aussen hin. Nur so kann ich mich selbst finden und mit mir selbst in Einklang leben, dass ist zugleich ausserhalb meiner selbst versetzt werde. Sich selbst finden vollzieht sich, gemäss den Evangelien, immer nur als sich selbst verlieren, und nur indem ich mich verliere, kann ich mich neu wieder finden. Dieses Öffnen nach aussen hin hat eine doppelte Ausrichtung: es gilt in Hinsicht auf die Mitmenschen und auf die 
Wirklichkeit, in der ich lebe. Deshalb ist wahre Frömmigkeit nicht abgesonderte, isolierte, welt- und wirklichkeitsfremde, sondern weltoffene, menschenfreundliche, gemeinschaftlich geteilte Frömmigkeit. Zugleich aber gilt die Öffnung in einem tieferen Sinne, nämlich in Bezug auf Gott als der Fremde, der ganz Andere, der mir erst Gehalt, Sinn, Identität schenkt. Das fromme Einüben dieser offenen Beziehung zu Gott hat ihren privilegierten Ort im Gebet als existentiellem Ringen mit dem Fremden.

\section{Zur Endlichkeit stehen}

Spiritualitäten faszinieren vielleicht deshalb so stark die Zeitgenossen, weil sie auf vielfältige Weise versprechen, den Menschen weg von sich selbst in weite, offene, tröstliche, lichte und warme Wirklichkeiten zu führen, wo er zur Ruhe kommen kann, Harmonie, Frieden erfahren kann. Sie entsprechen der menschlichen Sehnsucht nach Unendlichem, Unbegrenztem, lassen ihn seine Grenzen vergessen. Deshalb auch haben die neueren Spiritualitäten auch viel mehr Interesse für das Geistliche, das Seelische, als das Leibliche.

Demgegenüber muss hier betont werden, dass wahre Frömmigkeit gerade darin besteht, nüchtern und gelassen zur Endlichkeit zu stehen. Wenn auch die christliche Auffassung des Menschen Unendlichkeitsversuchungen gekannt hat, so geht doch ihr Grundzug in umgekehrte Richtung: Leiblichkeit, Zeitlichkeit und Sterblichkeit, und deshalb Begrenztheit gehören zur menschlichen Wirklichkeit, die es nicht abzulehnen, sondern anzunehmen gilt.

Das enthält auch eine ethische Komponente. Gewisse Frömmigkeitsströmungen können zu einem Enthusiasmus führen, der die Überzeugung stiftet, man könne und müsse nun die gesamte Welt ins Reine bringen, sie ein für allemal vom Bösen erlösen. Andere führen zu einem resignierten Rückzug aus der Welt: Von ihr ist nichts mehr zu erwarten, sie ist ohnehin verdorben und verloren. Wahre Frömmigkeit steht nüchtern und gelassen zwischen diesen zwei Extremen, setzt sich mit der Aufgabe auseinander, mit den begrenzten Mitteln, die einem zukommen, das Seine zu tun. In diesem Sinne sagte mal der südafrikanische Schriftsteller André Brink in einem seiner Romane, es gebe nur zwei gefährliche Torheiten, die, zu meinen, man könne nichts tun, und die, zu meinen, man könne alles tun.

\section{Witz und Geist}

Die im vorangegangenen Abschnitt erwähnte Nüchternheit und Gelassenheit hat ihren Grund in einem anderen Zug der Frömmigkeit, den ich hier mit dem Geist in Verbindung bringen möchte, doch im Sinne des französischen esprit. Es ist ja bei weitem nicht ausgeschlossen, dass diese Komponente auch etwas mit heiligem Geist zu tun hat! „Esprit“ haben, meint im Französischen Humor haben. Eine ähnliche Verbindung stellt im Deutschen das Begriffspaar „Witz und Geist" her, wie auch im Englischen wit and spirit.

Humor, als geistig-geistliche Einstellung, gehört zur Frömmigkeit. Er kennzeichnet die innere Distanz, die es dem Menschen erlaubt, die Dinge des Lebens gelassen und nüchtern wahrzunehmen und aufzunehmen. In diesem 
Sinne ist er Ausdruck der christlichen Freiheit. Er nimmt sich die Freiheit, immer wieder zu fragen, abzuwägen, was denn einem auch wirklich frommt. Um es mit Paulus zum Ausdruck zu bringen: „Prüfet alles, und behaltet das Rechte." (1. Thess 5, 21)

So schafft Humor immer wieder die Verbindung von Freiheit und Frömmigkeit, als alltägliche Lebensaufgabe.

\section{Diesseits des Denkens}

Kritische Frömmigkeit lebt von einer Gewissheit, die sich selbst nicht vortäuscht, alle Anfechtungen schon überwunden zu haben. In Hinsicht auf die Auseinandersetzung mit dem Denken heisst das: sie ist nicht schon jenseits, sondern immer noch diesseits des Denkens. Das gilt in christlicher Perspektive auch für die Beziehung zur Theologie: Gerade darin, dass sie sich der theologischen Reflexion aussetzt, ist sie kritische Frömmigkeit. Doch gilt dies auch in umgekehrter Richtung: Die Theologie ist nicht schon jenseits von der Frömmigkeit, sie hinter sich lassend. Der gelebte Glaube geht ihr voran, aber er begleitet sie auch stets als kritische Instanz. Die Frömmigkeit stellt der Theologie immer wieder die kritische Frage: was frommst du, was nützt du dem Menschen? In dieser Sorge um die Lebenserfahrung, um den gelebten Glauben ist die Frömmigkeit wahrhaft kritische Frömmigkeit, die stets bereit ist, den Widerstreit mit dem Denken auszuhalten. Doch nur wenn die theologische Reflexion diesen Widerstreit wahrnimmt, sich ihm aussetzt, wird sie auch zu einer wahrhaft kritischen Theologie. Theologie und Frömmigkeit können so aneinander lernen und reifen. und Fundamentaltheologie, an der Universität Zürich. 